\title{
Neoadjuvant Chemoradiotherapy and Total Mesorectal Excision in the Management of Locally Advanced Rectal Carcinoma - The PGH CRPoCan Study Group Experience 2008-2009
}

\author{
Henri S. Co, ${ }^{1}$ Marie Dione P. Sacdalan, ${ }^{2}$ Marc J. Lopez,,$^{2}$ Irisyl O. Real, ${ }^{3}$ Mark C. Ang, ${ }^{4}$ \\ Edilberto V. Fragante, ${ }^{1}$ Manuel T. Roxas, ${ }^{2}$ Dennis L. Sacdalan ${ }^{3}$ and Andrew D. Dimacali ${ }^{4}$

\begin{abstract}
${ }^{1}$ Section of Radiation Oncology, Department of Radiology, College of Medicine and Philippine General Hospital, University of the Philippines Manila ${ }^{2}$ Division of Colorectal Surgery, Department of Surgery, College of Medicine and Philippine General Hospital, University of the Philippines Manila ${ }^{3}$ Section of Medical Oncology, Department of Medicine, College of Medicine and Philippine General Hospital, University of the Philippines Manila ${ }^{4}$ Department of Laboratories, Philippine General Hospital, Manila
\end{abstract}

\begin{abstract}
Introduction. The use of neoadjuvant chemoradiotherapy (CRT) and total mesorectal excision (TME) has shown promising results in the management of locally advanced rectal carcinoma, and is associated with improvement in local control, disease free survival (DFS) and overall survival (OS). However, these clinical endpoints cannot be properly assessed due to poor follow up among many patients. Other endpoints such as negative circumferential resection margins (CRM), pathologic complete response ( $p C R$ ) and sphincter-preserving surgery (SPS) may serve as indirect means of assessing successful treatment. This study reports the experience of the Philippine General Hospital (PGH) Colorectal Polyp and Cancer (CRPoCan) Study Group in using neoadjuvant CRT and TME in the management of locally advanced rectal carcinoma, towards quality care.
\end{abstract}

Methods. The Integrated Surgical Information System (ISIS) database of the Department of Surgery, PGH was queried for rectal cancer patients with pretreatment clinical stage II and III disease that underwent neo-adjuvant CRT followed by TME between January 2008 and December 2009. The final surgical pathology reports of the subjects were reviewed for treatment response. Response was categorized as: (1) positive or negative CRM; and (2) with or without PCR. The study assessed whether SPS was done.

Results. Of 140 potential neoadjuvant CRT patients followed by TME, 82 patients completed the treatment. Thirty two of the patients who completed treatment (39\%) were eligible since the other 50 patients (61\%) had no post-operative histopathology results. Among those eligible, 10 patients (31\%) had pCR. Only 1 patient had a positive CRM. Of the 14 patients whose tumor

Presented at the 65th Annual Convention of the Philippine College of Radiology, February 2013, SMX Convention Centre, Pasay City.

Corresponding author: Dennis L. Sacdalan, MD, MCMMO, PhD (cand) Mol Bio Section of Medical Oncology

Department of Medicine

Philippine General Hospital

University of the Philippines Manila

Taft Avenue, Ermita, Manila 1000 Philippines

Tele/Fax: +632 5263775

Email: dennis.sacdalan@gmail.com distance was $\leq 5 \mathrm{~cm}$ from the anal verge, only 1 patient underwent SPS. The small sample size was mainly attributed to low resources for treatment. Non-availability of post-operative histopathology results was due to poor record keeping.

Conclusion. The PGH CRPoCan Study Group's use of neoadjuvant CRT followed by TME for locally advanced rectal carcinoma has resulted in acceptable numbers of $p C R$ and clear CRM but has not translated into an increased number of SPS. Despite the limitations of the study, the institutionalization of the multidisciplinary team in the PGH CRPoCan Study Group and the implementation of the ISIS database program are considered the first steps towards quality health care.

Key Words: neoadjuvant chemoradiotherapy, total mesorectal excision, rectal carcinoma, quality care

Introduction

The use of neoadjuvant chemoradiotherapy (CRT) and total mesorectal excision (TME) has shown promising results in the management of locally advanced rectal carcinoma, and is associated with improvement in local control, disease free survival (DFS) and overall survival (OS). However, many patients who undergo multidisciplinary treatment are usually lost to follow up. Hence local control, DFS, and OS cannot be assessed properly. Other endpoints such as negative circumferential resection margin (CRM), pathologic complete response $(\mathrm{pCR})$ and sphincter-preserving surgery (SPS) may serve as indirect means of evaluating successful treatment and as indicators of quality of care.

Studies have shown that a positive CRM is associated with a high risk of local recurrence, distant metastasis and poor OS after TME for rectal cancer. ${ }^{1}$ On the other hand, pCR is associated with decreased local recurrence, improved recurrence-free survival, and increased SPS. ${ }^{2}$ SPS is considered a surrogate for surgical quality, and is of utmost importance in maintaining the quality of life of a patient. ${ }^{3}$

This study reports the experience of the Colorectal Polyp and Cancer (CRPoCan) Study Group of the Philippine General Hospital (PGH) in using neoadjuvant CRT and TME in the management of locally advanced rectal carcinoma. 


\section{Methods}

The Integrated Surgical Information System (ISIS) database of the PGH Department of Surgery was queried for rectal cancer patients who had pre-treatment clinical stage II and III disease and who underwent neoadjuvant CRT between January 2008 and December 2009. Only patients who completed full course neoadjuvant CRT followed by TME were included. Exclusion criteria included the presence of concomitant inflammatory bowel disease, hereditary colorectal cancer syndrome, second-primary malignancies, and need for urgent surgery.

The preoperative clinical stage of each subject was based on endorectal ultrasound, CT scan, MRI, physical examination, or a combination of these. All patients received pelvic radiation via multiple-field technique to reach 5000 cGy concurrently with fluoropyrimidine-based chemotherapy. Neoadjuvant chemotherapy was delivered as either: bolus infusion of 5-fluorouracil (5-FU) $425 \mathrm{mg} / \mathrm{m}^{2} / \mathrm{d}$ with leucovorin $20 \mathrm{mg} / \mathrm{m}^{2} / \mathrm{d}$ given for 5 consecutive days during weeks 1 and 5 of radiation treatment; or oral capecitabine $825 \mathrm{mg} / \mathrm{m}^{2}$ twice daily for 5-7 days per week taken during radiation periods. This was a retrospective descriptive study and therefore regimens were expected to vary.

Furthermore, all cases have undergone "optimal" rectal resection with the technique of sharp mesorectal excision. This technique involves dissection in the areolar plane between the fascia propria (visceral fascia) of the rectum and the parietal pelvic fascia. For upper-rectal tumors, the rectum and mesorectum are divided $5 \mathrm{~cm}$ distal to the caudal tumor edge. For tumors of the mid and distal rectum, a TME is performed.

The final surgical pathology reports of the subjects were reviewed for treatment response. They were categorized as: (1) positive or negative CRM; and (2) with or without pCR. Assessment of anal sphincter function was done clinically.

Patient medical records and the colorectal cancer clinical database were reviewed to collect demographic data such as age, sex, distance from the anal verge, histologic type, tumor differentiation, CEA, clinical stage, chemotherapy regimen, interval time to surgery, type of resection, ypT stage, ypN stage, and circumferential resection margin. The study did not assess local control, DFS and OS.

Continuous data were summarized using mean, median, standard deviation, and range. Categorical data were summarized using frequency tables.

The study was conducted in accordance with the guidelines of the Helsinki Declaration. The study was submitted to the Expanded Hospital Research Office (EHRO) of PGH for technical review and approval prior to initiation of the study. Codes were used to mask the identities of the patients. Data gathered were reported as group data.

\section{Results}

From 01 January 2002 to 31 December 2009, 418 rectal cancer patients underwent external beam radiation therapy at the PGH Section of Radiation Oncology. Of the 418 patients, 373 patients $(89.23 \%)$ were able to complete the prescribed radiation therapy sessions. Of the 45 patients $(10.76 \%)$ who were unable to complete the prescribed radiation therapy sessions, 26 patients $(58 \%)$ were in the neoadjuvant CRT group while 19 patients (42\%) were in the adjuvant CRT group.

There was a steady increase in the number of rectal cancer patients treated with external beam radiation therapy from 2002 to 2009. During this period, there was a gradual increase in the number of patients who underwent neoadjuvant CRT and a corresponding decrease in the number of patients treated with adjuvant CRT (Table 1).

Table 1. Census of rectal cancer patients treated with external beam radiation therapy at the PGH Section of Radiation Oncology from 2002-2009.

\begin{tabular}{ccccc}
\hline Year & $\begin{array}{c}\text { Neoadjuvant } \\
\text { RT }\end{array}$ & $\begin{array}{c}\text { Neoadjuvant } \\
\text { CRT }\end{array}$ & $\begin{array}{c}\text { Adjuvant } \\
\text { CRT }\end{array}$ & TOTAL \\
\hline 2002 & $3(13 \%)$ & $3(13 \%)$ & $17(74 \%)$ & 23 \\
2003 & $2(7 \%)$ & $2(7 \%)$ & $26(86 \%)$ & 30 \\
2004 & $4(13 \%)$ & $2(6 \%)$ & $26(81 \%)$ & 32 \\
2005 & $5(12 \%)$ & $9(21 \%)$ & $28(67 \%)$ & 42 \\
2006 & $9(17 \%)$ & $11(20 \%)$ & $34(63 \%)$ & 54 \\
2007 & $17(29 \%)$ & $11(19 \%)$ & $30(52 \%)$ & 58 \\
2008 & $19(21 \%)$ & $42(48 \%)$ & $27(31 \%)$ & 88 \\
2009 & $9(10 \%)$ & $60(66 \%)$ & $22(24 \%)$ & 91 \\
TOTAL & $68(16 \%)$ & $140(34 \%)$ & $210(50 \%)$ & 418 \\
\hline
\end{tabular}

Of the eighty two (82) patients who completed neoadjuvant CRT from 2008 to 2009, the data of only 32 patients (39\%) were eligible since the other 50 patients $(61 \%)$ had no post-operative histopathology results. The characteristics of eligible patients who underwent neoadjuvant CRT followed by total mesorectal excision are in Table 2.

\section{Patient and Tumor Characteristics}

The population had a mean age of 50 years, with half of the population $<50$ years old. There were $53 \%$ males and $47 \%$ female patients. Majority of the tumors were seen in the lower $(44 \%)$ and mid (31\%) rectum. This histology of $56 \%$ was well-differentiated adenocarcinoma. Mean CEA was $22.66 \mathrm{ng} / \mathrm{mL}$. Fifty six percent of the patients had clinical stage IIIB.

\section{Treatment Characteristics}

The patients received radiation therapy with a mean dose of 50 Gy. Seventy five percent received 5-FU and leucovorin intravenously. The median time to surgery was 8.65 weeks. Most patients had an anterior resection (38\%) or an anterior transanal resection (33\%). Of the 14 patients whose tumor distance was $\leq 5 \mathrm{~cm}$ from the anal verge, only 1 patient had SPS. 
Table 2. Profile of eligible rectal cancer patients

\begin{tabular}{|c|c|}
\hline CHARACTERISTIC & $\begin{array}{c}\text { NEOADJUVANT } \\
\text { CRT } * \text { TME } \\
(\mathrm{N}=32)\end{array}$ \\
\hline \multicolumn{2}{|l|}{ Age - years } \\
\hline Median & 50 \\
\hline Range & $28-71$ \\
\hline \multicolumn{2}{|l|}{ Sex - no. (\%) } \\
\hline Male & $17(53 \%)$ \\
\hline Female & $15(47 \%)$ \\
\hline \multicolumn{2}{|c|}{ Distance of tumor from the anal verge - no. $(\%)$} \\
\hline$\leq 5 \mathrm{~cm}$ & $14(44 \%)$ \\
\hline$>5-\leq 10 \mathrm{~cm}$ & $10(31 \%)$ \\
\hline$>10 \mathrm{~cm}$ & $1(3 \%)$ \\
\hline Unknown & $7(22 \%)$ \\
\hline \multicolumn{2}{|l|}{ Histologic type - no. (\%) } \\
\hline Adenocarcinoma & $27(84 \%)$ \\
\hline Mucinous & $4(13 \%)$ \\
\hline Signet Ring & $1(3 \%)$ \\
\hline \multicolumn{2}{|l|}{ Differentiation - no. (\%) } \\
\hline Well differentiated & $18(56 \%)$ \\
\hline Moderately differentiated & $4(12.5 \%)$ \\
\hline Poorly differentiated & $6(19 \%)$ \\
\hline Unknown & $4(12.5 \%)$ \\
\hline \multicolumn{2}{|l|}{ CEA } \\
\hline Median & 22.66 \\
\hline Range & $0.85-118.5$ \\
\hline \multicolumn{2}{|l|}{ Clinical stage - no. $(\%)$} \\
\hline $2 \mathrm{~A}$ & $4(13 \%)$ \\
\hline 2B & $2(6 \%)$ \\
\hline $3 \mathrm{~A}$ & $6(19 \%)$ \\
\hline 3B & $18(56 \%)$ \\
\hline Unknown & $2(6 \%)$ \\
\hline \multicolumn{2}{|l|}{ RT dose - Gy } \\
\hline Median & 50 \\
\hline Range & $50-50.4$ \\
\hline \multicolumn{2}{|l|}{ Chemotherapy regimen - no. (\%) } \\
\hline 5-FU + Leucovorin & $24(75 \%)$ \\
\hline Capecitabine & $3(9 \%)$ \\
\hline Unknown & $5(16 \%)$ \\
\hline \multicolumn{2}{|l|}{ Interval time to surgery - weeks } \\
\hline Median & 8.65 \\
\hline Range & $3-42$ \\
\hline \multicolumn{2}{|l|}{ Type of resection - no. (\%) } \\
\hline Anterior resection & $12(38 \%)$ \\
\hline Abdominoperineal resection & $7(22 \%)$ \\
\hline Hartmann's & $3(9 \%)$ \\
\hline Anterior Transanal Resection & $10(31 \%)$ \\
\hline \multicolumn{2}{|l|}{ Histopathological finding - no. (\%) } \\
\hline \multicolumn{2}{|l|}{ Tumor } \\
\hline ур т0 & $10(31 \%)$ \\
\hline yp T1 & $1(3 \%)$ \\
\hline ур Т2 & $7(22 \%)$ \\
\hline yp Т3 & $9(28 \%)$ \\
\hline yp T4 & $5(16 \%)$ \\
\hline \multicolumn{2}{|l|}{ Nodes } \\
\hline Total examined - no. & \\
\hline Mean & 9.78 \\
\hline Range & $0-24$ \\
\hline yp No & $27(85 \%)$ \\
\hline yp N1 & \\
\hline A & $1(3 \%)$ \\
\hline B & $1(3 \%)$ \\
\hline $\mathrm{C}$ & $0(0 \%)$ \\
\hline yp N2 & \\
\hline A & $2(6 \%)$ \\
\hline B & $1(3 \%)$ \\
\hline Circumferential resection margin - nc & \\
\hline R0 & $26(81 \%)$ \\
\hline R1 & $0(0 \%)$ \\
\hline $\mathrm{R} 2$ & $1(3 \%)$ \\
\hline Unknown & $5(16 \%)$ \\
\hline
\end{tabular}

\section{Pathologic Characteristics}

Of the 32 eligible patients who underwent neoadjuvant CRT followed by TME, 10 patients (31\%) achieved pCR. The mean number of lymph nodes harvested was $9.78,85 \%$ of which were negative for metastasis. Only 1 patient had a positive CRM.

\section{Discussion}

From 2002 to 2009, there was a gradual increase in the number of patients referred from both within and outside PGH for the management of rectal cancer. During the same time period, there was also a gradual increase in the number of patients treated with neoadjuvant CRT followed by TME, and a corresponding decrease in the number of patients treated with adjuvant CRT. The sudden rise in the number of patients receiving neoadjuvant CRT in 2005 and 2008 reflected two important milestones in local rectal cancer treatment, namely the publication of the German Rectal Cancer Study in $2004^{4}$ and the introduction of the PGH CRPoCan Study Group in late 2007.

Most of the patients receiving neoadjuvant CRT followed by TME were referred from within the hospital while most of the patients receiving adjuvant CRT after nonTME surgeries were referred by other hospitals from around the country. Since PGH caters to mostly indigent Filipinos who cannot afford basic health care or medical insurance, financial constraints was identified as the major reason for incomplete treatment in $10.76 \%$. Other reasons may be due to morbidity or mortality from the treatment or the disease.

Only 32 out of 82 patients (39\%) who completed neoadjuvant CRT from 2008 to 2009 had their post-operative histopathology results available and were eligible for the study. This low turnout could be attributed to poor record keeping and poor follow up due to financial constraints, morbidity or mortality.

\section{Patient and Tumor Characteristics}

Half of the population is composed of patients $<50$ years old and most of the tumors are located at the lower to mid rectum. These patients are the ideal population that would benefit the most from having neoadjuvant CRT followed by TME as this treatment approach gives better local control and the chance to achieve SPS. Though most patients have a favorable histology of well-differentiated adenocarcinoma, they have locally advanced disease, which carries a poorer prognosis.

\section{Treatment Characteristics}

Most of the patients received 5-FU and leucovorin intravenously concurrently with RT since it was the most affordable regimen for indigent patients. Eighty one percent of the patients had their surgery within the recommended 510 week period from the last RT session, with some surgeries being delayed due to financial constraints. The type of 
resections done on the patients could be correlated with the location of the tumor from the anal verge. However, only 1 patient $(7 \%)$ had SPS compared to published literature citing a $39-58 \%$ SPS rate. ${ }^{4,5,6}$

\section{Pathologic Characteristics}

This study showed that $31 \%$ of patients had a pCR, as compared to the published reports citing only a pCR rate of $8-19.2 \% .4,5,7,8$ This result may be falsely high since $61 \%$ of the patients who had neoadjuvant CRT followed by TME had no post-operative histopathology results. Only $37.5 \%$ of patients had the recommended $\geq 12-14$ lymph nodes harvested from the TME specimen. This may be due to poor surgical technique, poor processing by the pathologist, or poor response to treatment. Eighty five percent of the postoperative specimens were negative for lymph node metastasis, which was higher compared to published reports of $66.7-75 \%$.

Only 1 patient had a positive CRM, while the rest were declared to have "clear" margins. This was comparable to published literature of $4-9.9 \% .5,7$ However, measurement of the proximal, distal and circumferential margins in millimeters was not done, hence objective determination of whether there were close margins or R1 resection was limited. There was also no mention of post-RT changes or lymphatic invasion, which is a risk factor for recurrence, in any of the post-operative specimens by the pathologist.

\section{Conclusion}

The PGH CRPoCan Study Group's use of neoadjuvant CRT followed by TME for locally advanced rectal carcinoma resulted in acceptable numbers of pCR and clear CRM. The treatment approach did not translate into increased numbers of SPS most probably because of the very small sample size of 32 from a potential patient population of 140 who underwent neoadjuvant CRT. Ineligibility to inclusion into the study was due to non-completion of the prescribed radiation therapy sessions and the absence of post-operative histopathology results. Inability to complete treatment was mainly a reflection of the limitations in an out-of-pocket health care setting, as well as morbidity or mortality due to the treatment or the disease. Non-availability of postoperative histopathology results was due to poor record keeping.

Record keeping must be of high quality if we were to convert data provided by the huge volume of PGH patients into more meaningful research. Efforts to maximize learning from patients are needed in order to improve health care as provided by the Universal Health Care Act of 2013, which calls for adequate funding for the proper treatment of patients. It must be noted however that the first steps towards quality care, specifically for colorectal cancer, have already been established through the institutionalization of the multidisciplinary team in the PGH CRPoCan Study Group, and the use of ISIS database program.

\section{References}

1. Wibe A, Rendedal PR, Svensson E, et al. Prognostic significance of the circumferential resection margin following total mesorectal excision for rectal cancer. Br J Surg. 2002; 89(3):327-34.

2. Kalady MF, de Campos-Lobato LF, Stocchi L, et al. Predictive factors of pathologic complete response after neoadjuvant chemoradiation for rectal cancer. Ann Surg. Aug; 250(4):582-9.

3. Temple LK, Romanus D, Niland J, et al. Factors associated with sphincter-preserving surgery for rectal cancer at national comprehensive cancer network centers. Ann Surg. Aug; 250(2):260-7.

4. Sauer R, Becker H, Hohenberger W, et al. Preoperative versus postoperative chemoradiotherapy for rectal cancer. N Engl J Med. 2004; 351(17):1731-40.

5. Bujko K, Nowacki MP, Nasierowska-Guttmejer A, Michalski W, Bebenek M, Kryj M. Long-term results of a randomized trial comparing preoperative short-course radiotherapy with preoperative conventionally fractionated chemoradiation for rectal cancer. Br J Surg. 2006; 93(10):1215-23.

6. Bosset JF, Collette L, Calais G, et al. Chemotherapy with preoperative radiotherapy in rectal cancer. N Engl J Med. 2006; 355(11):1114-23.

7. Gérard J, Conroy T, Bonnetain F, et al. Preoperative radiotherapy with or without concurrent fluorouracil and leucovorin in T3-4 rectal cancers: results of FFCD 9203. J Clin Oncol. 2006; 24(28):4620-5.

8. Roh MS, Colangelo LH, O'Connell MJ, et al. Preoperative multimodality therapy improves disease-free survival in patients with carcinoma of the rectum: NSABP R-03. J Clin Oncol. 2009; 27(31):512430 . 